\title{
Construcción y validación de la escala de tipo de espectador de violencia entre pares (TEVEP)
}

\author{
Construction and validation of scale type of violence pair viewer (TEVEP)
}

\author{
Alberto Quintana P.1, Willam Montgomery U., Carmela Malaver S., Sergio Dominguez L., \\ Gabriela Ruiz S., Nayú García Z., Eduardo Moras R. \\ Universidad Nacional Mayor de San Marcos, Lima, Perú \\ (RECIBIDO 05-08-2014, AcEPTADo 20-10-2014)
}

\begin{abstract}
RESUMEN
Se presenta el estudio de las propiedades psicométricas de un instrumento para identificar tres tipos de espectadores reconocibles en situaciones de violencia entre pares. La muestra de estudio estuvo conformada por 480 adolescentes, de 14 a 18 años (media $=16.17$ ), de nivel socioeconómico medio-bajo y bajo, pertenecientes a instituciones educativas de Lima Metropolitana. La escala tipos de espectadores de violencia entre pares (TEVEP) se elaboró mediante una metodología cualitativa/cuantitativa, mediante cuatro fases, siendo el objetivo de este trabajo la cuarta fase consistente en un análisis factorial confirmatorio hipotetizando un modelo trifactorial (Espectador pro social, espectador indiferente-culpabilizado y espectador antisocial). En conclusión, luego de un análisis exploratorio intermedio, el Tevep cuenta con propiedades psicométricas adecuadas para promover estudios que consoliden tales indicadores.
\end{abstract}

Palabras clave: Violencia entre pares, tipos de espectadores, validez, análisis factorial confirmatorio, TEVEP.

\begin{abstract}
The aim of this paper is the study of the psychometric properties of an instrument to identify three types of viewers that can be found in situations of violence among peers. The study sample consisted of 480 adolescents, aged 14 to 18 years (mean $=16.17)$, middle - low and low socioeconomic status and of educational institutions from Lima. Scale Types Viewers Violence Peer (STVVP) was developed through a qualitative/quantitative methodology consists of four phases, with the aim of this work the fourth phase was aimed at the implementation of a confirmatory factor analysis hypothesizing one three-factor model (Prosocial Spectator, indifferent-feels guilty Spectator, and Antisocial Spectator). In this regard, based on the evidence found, after an intermediate exploratory analysis, STVVP has adequate psychometric properties to promote studies that consolidate such indicators.
\end{abstract}

Keywords: Peer violence, types of viewers, validity, confirmatory factor analysis, TEVEP.

1 Docente principal de la Facultad de Psicología de la UNMSM, Perú. E-mail: albertolqp@hotmail.com 


\section{INTRODUCCIÓN}

Desde hace varias décadas, el mundo progresivamente ha observado cómo la violencia se ha convertido en tema principal y encabezado de muchos titulares, en los periódicos y otros medios de comunicación masiva como la radio, televisión, internet, mostrándose imágenes sin censura, noticias sobre homicidios, parricidios, conflictos internacionales, guerras internas y todo tipo de violencia. Al respecto, en sus inicios los espectadores, en su gran mayoría, veían con alarma estas manifestaciones de violencia y se preguntaban si acaso el mundo se dirigía a su inminente destrucción. Sin embargo hoy por el contrario, pareciera que han incorporado este fenómeno como parte de su diario devenir, ante la exposición continua de información sobre violencia ocurrida en las calles, en los hogares y también en las escuelas. Es en este último ámbito, que muchos investigadores de diferentes ciencias en nuestro país, han tomado interés, hace pocos años, por esta problemática que recibe el nombre de bullying o violencia entre pares, una forma de intimidación y maltrato entre escolares (Avilés, 2006).

La escuela constituye un ambiente de fuerte incidencia y hasta de cultivo de ciertas actitudes agresivas debido al carácter aislante o competitivo de muchas de sus actividades. Dichas actitudes, aun cuando no llegasen a convertirse en conducta antisocial delictiva, son motivo de preocupación para los padres, maestros y pares.

Al respecto, la Comisión Nacional para el Desarrollo y Vida sin Drogas en su II Estudio Nacional de Prevención y Consumo de Drogas en Estudiantes de Secundaria, reveló que uno de cada cuatro escolares, es decir, el 24,3\%, ha sido víctima de una o más agresiones físicas en lo que va de su vida escolar. El 15,3\% señala que el agresor o los agresores fueron compañeros de clase, mientras el 9,9\% manifiesta que fue un profesor, auxiliar $\mathrm{u}$ otra autoridad escolar, hecho que va marcando, sin lugar a dudas, una influencia en las relaciones de convivencia entre los alumnos.

En nuestro medio, al igual que en el resto del mundo (Quintana \& Ruiz, 2013), las investigaciones empíricas sobre el tema de bullying o violencia entre pares centran su atención en la relación víctima-victimario, mientras que dejan de lado, en la mayoría de las veces, a un actor que puede jugar un papel, tanto de reforzador de la conducta violenta como de inhibidor de ésta: el espectador. Cuando hablamos de espectador, nos referimos a aquel alumno que observa, activa o pasivamente, las agresiones del victimario contra la víctima. Las razones del espectador para actuar o no pueden ser variadas, desde el temor a no entrometerse en el asunto para "no ganarse un pleito", hasta el espectador que simplemente se acostumbró a ver las agresiones y se mantiene indiferente bajo la creencia de que "ya nada se puede hacer". Dentro de estos tipos de espectadores, también podemos encontrar a aquellos que, de una manera directa o indirecta, se enfrentan al victimario para poder evitar los abusos que éste comete con la víctima.

En referencia a estas conceptualización nuestro equipo de investigación (Quintana, Montgomery, Malaver, 2009) elaboró una versión preliminar de 30 ítems del presente instrumento, con la cual se han llevado a cabo una serie de investigaciones empíricas referentes, por un lado, a los modos de afrontamiento y conducta resiliente; por otro lado a estilos explicativos y habilidades para la gestión de 
negociación de conflictos; y, finalmente, a la capacidad de disfrute y percepción del apoyo comunitario; todo ello utilizando muestras de adolescentes espectadores de episodios de violencia entre pares (Quintana, Montgomery, Malaver \& Ruiz, 2009, 2010, 2011), obteniéndose valiosos datos y conclusiones. Por ejemplo, se ha verificado que el espectador de tipo pro-social utiliza estrategias de afronte para solucionar el problema de tipo instrumental, de planificación, de apoyo entre otros, es más resiliente, posee en mayor grado habilidades para la gestión de conflictos interpersonales, tiende a explicar sus éxitos en las relaciones interpersonales gracias a su propio esfuerzo y habilidad, tiene más capacidad de disfrute, sentimiento de bienestar subjetivo y percepción subjetiva de apoyo comunitario, sin embargo habiéndose realizado estos hallazgos con una versión preliminar ahora se requiere reproducir estos análisis a fin de consolidar de una forma más rigurosa el presente instrumento.

Ello evidencia la importancia de tener un instrumento que detecte en el contexto escolar los tipos de espectadores, de manera que nos permita considerarlos actores, con un rol activo en la intervención frente a los abusos cometidos por los bullies. Que éstos últimos observen que su entorno rechaza sus actitudes y además, tanto el espectador como la víctima formen alianzas que contribuyen a que estas conductas no adecuadas se minimicen o hasta desaparezcan. De ahí la importancia de difundir el instrumento Tevep, el cual, en base a un cuestionario tipo Likert de 26 preguntas, nos ayuda a reconocer los tres tipos de espectadores que según Monjas y Avilés (2006) se encuentran en este tipo de violencia.

\section{Conceptualización de las variables estudiadas}

\section{Violencia entre pares}

Existen muchas definiciones para lo que conocemos como bullying o violencia entre pares. En este artículo suscribiremos la definición de Avilés (2006), cuando conceptualiza la violencia entre pares como la intimidación y el maltrato entre escolares de forma repetida y mantenida en el tiempo, siempre lejos de la mirada de los adultos/as, con la intención de humillar y someter abusivamente a una víctima indefensa por parte de un abusón o grupo de matones a través de agresiones físicas, verbales y/o sociales con resultados de victimización psicológica y rechazo grupal. El resultado de este tipo de interacción interpersonal refleja diversidad en los perfiles de los participantes (Avilés \& Monjas, 2005): de víctima (pasiva, provocativa, reactiva, agresiva), de agresor (seguro, ansioso, secuaz), de agresor victimizado (en ocasiones agresor y en ocasiones víctima) y de espectadores (pares culpabilizados, indiferentes o amorales y adultos).

\section{Tipos de espectador}

Los espectadores son aquellos que contemplan las agresiones sin poder o querer evitarlas. Se ha argumentado que esto es producto del miedo a ser incluidos dentro del círculo de la victimización y convertirse en blanco de agresiones (Lecannelier, 2006, cit. por Varela \& Tijmes, 2008; Olweus, 1998). Sin embargo, es pertinente 
resaltar que su presencia como testigos puede ser determinante en el episodio de bullying, al estimular o inhibir las acciones del agresor (Trautman, 2008).

Entre los espectadores es posible distinguir tres tipos, a saber:

a) Espectador indiferente-culpabilizado, observa la agresión; pero considera que no es un asunto de su competencia porque a él no le toca. Puede incluso considerar injusta la agresión, sentirse afectado por lo que sucede; pero no interviene por temor a ser la próxima víctima.

b) Espectador amoral, justifica la agresión porque considera que, en la vida, unos son más fuertes y otros débiles o porque la víctima lo provocó.

c) Espectador pro-social, enfrenta activamente la situación, confrontando al victimario y/o solicitando ayuda de otros compañeros o docentes (Monjas \& Avilés, 2006).

\section{MÉTODO}

\section{Participantes}

Para estudiar las propiedades psicométricas de la escala en esta última fase se trabajó con una muestra no probabilística e intencional constituida por 480 adolescentes de ambos sexos que cursan el nivel secundario, cuyo rango de edad varía entre los 14 y 18 años (media $=16.17$ ), de nivel socioeconómico medio-bajo pertenecientes a instituciones educativas de gestión tanto estatal como no estatal ubicadas en Lima Metropolitana.

Tabla 1. Características sociodemográficas de la muestra

\begin{tabular}{ccc}
\hline & Frecuencia & Porcentaje \\
\hline Sexo & 254 & 52,9 \\
Varón & 221 & 46,0 \\
Mujer & 5 & 1,0 \\
MD & & \\
Edad & 13 & 2,7 \\
14 & 114 & 23,8 \\
15 & 181 & 37,7 \\
16 & 119 & 24,8 \\
17 & 49 & 10,2 \\
18 & 4 &, 8 \\
MD & & \\
Socioeconómico & 444 & 92,5 \\
Bajo & 36 & 7,5 \\
Medio-Bajo & & 40,4 \\
Grado Escolar & 194 & 286 \\
4to & 286 & \\
5to & & \\
\hline
\end{tabular}

$\mathrm{MD}=$ Datos perdidos 


\section{Instrumento y procedimiento}

La escala de tipos de espectadores de violencia entre pares (Tevep) fue diseñada y administrada para determinar los tipos de espectadores en la violencia entre pares. Está finalmente constituida por 26 reactivos autoadministrados. Las respuestas a los reactivos se evalúan en un formato tipo Likert que incluye cuatro opciones, donde $\mathrm{N}=$ Nunca, $\mathrm{AV}=$ Alguna Vez, $\mathrm{CS}=$ Casi Siempre y $\mathrm{S}=$ Siempre. A cada opción se le asigna un valor de $0,1,2$ y 3 puntos, respectivamente. Las instrucciones del instrumento se explican claramente para que el sujeto evaluado no tenga inconvenientes en la elección de su respuesta.

En el anexo 1 se presenta la versión definitiva resultante de los análisis realizados.

\section{Técnica de recolección de datos}

Para la construcción de la escala, en esta última fase se aplicó el cuestionario piloto constituido por 5 preguntas introductorias no calificables y 30 enunciados indicativos de los tipos de espectadores previstos, a una muestra de 480 participantes, a quienes se les preguntó además algunos datos demográficos (sexo, edad, nombre de la institución educativa, grado escolar), sometiéndose los resultados a las pruebas estadísticas que permitieron obtener la versión final de 26 ítems (Anexo 1) y sus datos de confiabilidad y validez.

\section{Procedimientos}

Como ya se adelantó líneas atrás para la construcción de la Escala de Tipos de Espectadores de Violencia entre Pares (TEVEP) se siguieron las siguientes fases:

Primera fase: Elaboración y validación de criterio de expertos de las cinco preguntas de los encabezados que componen la escala, presentándolas como preguntas abiertas en un estudio piloto a aproximadamente 400 estudiantes de educación secundaria entre 14 y 18 años. Las respuestas dadas por estos adolescentes, que permiten identificar emociones, acciones y creencias, fueron sometidas a un análisis de contenido, con el objetivo de discriminar las que eran distintivas de cada tipo de espectador (Monjas \& Avilés, 2006), desechando las ambiguas. Luego se seleccionaron las más frecuentes y representativas de cada tipo de espectador, realizándose una adaptación de su parafraseo, con otros adolescentes de secundaria, a fin de que las identifiquen como auto verbalizaciones de respuesta a las preguntas de los encabezados que ellos se harían así mismos (como ego-sintónicas).

Segunda fase: Elaboración de la primera versión psicométrica de la escala, constituida por 59 ítems. Aplicándola a 192 participantes y sometiéndola a una confiabilidad por la técnica de ítems pares e impares en la que se obtuvo una correlación positiva y significativa $(\mathrm{r}=.555, \mathrm{p}<.001)$, y validez por criterio de jueces obteniendo $\mathrm{V}$ de Aiken que oscilaron predominantemente entre 0.80 y 1.00 para cada ítem. 
Tercera fase: Diseño de una versión modificada de la escala, reducida a 30 ítems. Aplicándola a 480 participantes. En esta versión se incluía a manera de introducción la transcripción del encabezado y de los 5 primeros ítems del cuestionario para mejorar el conocimiento sobre uno mismo y sobre las relaciones con los demás, de Collell y Escudé (2003); del PRECONCIMEI como recurso para que el examinado se introduzca inadvertidamente al tema, por ello solo se califica el instrumento a partir del sexto ítem. (Quintana, Montgomery, Malaver \& Ruiz, 2009)

Cuarta fase: En este trabajo se presenta la cuarta fase en la elaboración del instrumento.

\section{Análisis Estadístico}

Una vez aplicados y codificados los datos en la base electrónica, se efectuaron los análisis estadísticos utilizando el programa LISREL para el análisis factorial confirmatorio, FACTOR para el análisis factorial exploratorio y un módulo para el cálculo del alfa ordinal (Domínguez, 2012).

\section{RESULTADOS}

\section{Análisis previo de datos}

Antes de proceder con el análisis factorial confirmatorio, se llevó a cabo un análisis de estadísticos descriptivos (Tabla 2), en el cual se constató que todos presentan indicadores de asimetría y curtosis adecuados, los cuales no exceden el rango +/- 1.5 (Pérez \& Medrano, 2010; Ferrando \& Anguiano-Carrasco, 2010).

\section{Análisis factorial confirmatorio}

Fue realizado el análisis factorial confirmatorio hipotetizando un modelo trifactorial (Espectador Pro social, Espectador indiferente-culpabilizado y Espectador Antisocial), de factores correlacionados, propuesto inicialmente a partir de las conceptualizaciones de Monjas y Avilés (2006). El método de estimación fue el de

Tabla 2. Estadísticos Descriptivos del Test de Espectadores de Violencia Entre Pares (Tevep)

\begin{tabular}{ccccc}
\hline & Media & $\begin{array}{c}\text { Desv. } \\
\text { Estándar }\end{array}$ & Asimetría & Curtosis \\
\hline Ítem 6 & 1.812 & .825 & -.363 & -.339 \\
Ítem 7 & 1.030 & .918 & .417 & -.835 \\
Ítem 8 & 1.205 & .982 & .277 & -.992 \\
Ítem 9 & 1.253 & 1.025 & .256 & -1.092 \\
Ítem 10 & 1.407 & .953 & .062 & -.932 \\
Ítem 11 & 1.951 & .916 & -.498 & -.629 \\
Ítem 12 & .812 & .884 & .909 & .045 \\
Ítem 13 & 1.044 & .921 & .529 & -.592 \\
Ítem 14 & 1.692 & .863 & -.309 & -.511
\end{tabular}


Alberto Quintana P.1, William Montgomery U., Carmela Malaver S., Sergio Dominguez L., Gabriela Ruiz S.

$\begin{array}{lcccc}\text { Ítem 15 } & .793 & .847 & .763 & -.296 \\ \text { Ítem 16 } & 1.327 & .979 & .132 & -1.013 \\ \text { Ítem 17 } & 1.481 & .887 & .030 & -.723 \\ \text { Ítem 18 } & 1.593 & .865 & -.131 & -.632 \\ \text { Ítem 19 } & 1.354 & .958 & .195 & -.895 \\ \text { Ítem 20 } & 1.470 & .842 & -.172 & -.615 \\ \text { Ítem 21 } & 1.259 & .945 & .274 & -.834 \\ \text { Ítem 22 } & 2.044 & .905 & -.721 & -.246 \\ \text { Ítem 23 } & 2.008 & .921 & -.650 & -.419 \\ \text { Ítem 24 } & 1.627 & .913 & -.111 & -.802 \\ \text { Ítem 25 } & 1.287 & .913 & .136 & -.845 \\ \text { Ítem 26 } & 1.479 & .924 & -.027 & -.842 \\ \text { Ítem 27 } & 1.156 & 1.009 & .326 & -1.065 \\ \text { Ítem 28 } & 1.880 & .959 & -.419 & -.820 \\ \text { Ítem 29 } & 1.949 & .887 & -.374 & -.760 \\ \text { Ítem 30 } & 1.017 & .995 & .532 & -.891 \\ \text { Ítem 31 } & 1.422 & .995 & .030 & -1.058 \\ \text { Ítem 32 } & 1.236 & .967 & .255 & -.941 \\ \text { Ítem 33 } & 2.167 & .936 & -.785 & -.498 \\ \text { Ítem 34 } & 1.268 & .918 & .332 & -.682 \\ \text { Ítem 35 } & 2.120 & .864 & -.725 & -.210\end{array}$

máxima verosimilitud, y aunque no se da el cumplimiento de supuesto de normalidad multivariada, presenta un adecuado indicador multivariado de asimetría $(\mathrm{G} 1=$ 162.309; $\mathrm{p}>$.05), pero no de curtosis (G2 = 1083.498; $\mathrm{p}<.05)$. Se analizaron las matrices de covarianzas, estableciendo la varianza de los factores a uno.

Asimismo, se usaron los indicadores de ajuste absoluto más frecuentes en investigación: $\chi^{2}$ (chi-cuadrado), Índice de Bondad de Ajuste (GFI), Índice Ajustado de Bondad de Ajuste (AGFI), Índice de Aproximación de la Raíz de Cuadrados Medios del Error (RMSEA), Índice de la Raíz del Cuadrado Medio del Residuo (RMR) y un indicador de incremento como el Índice de Ajuste Comparativo (CFI) el cual compara el modelo estimado (en este caso, el modelo de tres factores) con el modelo nulo que indique independencia entre las variables estudiadas (Ferrando \& Anguiano-Carrasco, 2010; Ruiz, Pardo \& San Martín, 2010; Sánchez \& Sánchez, 1998).

Tabla 3. Índices de Ajuste del Test de Espectadores de Violencia Entre Pares (Tevep)

\begin{tabular}{ccccccc}
\hline $\mathrm{X}^{2}$ & g.l. & CFI & GFI & AGFI & RMR & RMSEA \\
\hline $2065.3^{*}$ & 560 & .656 & .828 & .801 & .078 & .076 \\
\hline$*<.001$ & & & & & &
\end{tabular}

En base a los resultados, los valores obtenidos indican que los datos no se ajustan al modelo de tres factores propuesto por los autores del instrumento. En tal sentido, se necesitaría realizar un análisis exploratorio complementario a fin de conocer la estructura subyacente a los datos en la muestra estudiada (Bollen, 1989). 


\section{Análisis factorial exploratorio}

Se realizó un análisis factorial por Mínimos Cuadrados No Ponderados, extrayéndose tres factores en base a los supuestos teóricos del instrumento.

De forma previa, se examinó la intercorrelación de los ítems para realizar el análisis factorial. La matriz de correlaciones fue significativa $(\mathrm{p}<.01)$; se obtuvo un KMO de .778, valor considerado adecuado (Hair, Anderson, Tatham \& Black, 2004), y un test de esfericidad de Bartlett significativo $(\mathrm{p}<.01)$, lo cual da cuenta de la suficiente intercorrelación de los ítems. Cabe mencionar que se usó una matriz de correlaciones policóricas debido a la naturaleza ordinal de los reactivos.

Los tres factores extraídos explican el $33.50 \%$ de la varianza total de las puntuaciones. Para decidir por el tipo de rotación, sea ortogonal u oblicua, se optó por una rotación ortogonal (Weigthed Varimax) debido a que los supuestos teóricos indican independencia entre los tipos de espectadores que postula el instrumento.

Asimismo, fue evaluado el ajuste del modelo factorial resultante, el cual presenta un índice de bondad de ajuste (GFI, Ferrando \& Lorenzo-Seva, 1997) de .96, lo que indica que casi toda la varianza explicada a la que se refiere, se da en base al modelo de tres factores.

Tabla 4. Estructura factorial del Test de Espectadores de Violencia Entre Pares (TEVEP)

\begin{tabular}{|c|c|c|c|c|}
\hline & Ítems de la versión preliminar & F1 & $\mathrm{F} 2$ & F3 \\
\hline 12 & Me voy antes de que yo me convierta en lorna. & .437 & & \\
\hline 13 & No hago nada, para evitar que también a mí me molesten. & .395 & & \\
\hline 18 & $\begin{array}{l}\text { Que debo evitar esas situaciones, para no meterme en } \\
\text { problemas. }\end{array}$ & .370 & & \\
\hline 19 & Que si no las acusas, no te van a molestar. & .476 & & \\
\hline 20 & Que se lo merece porque seguro antes lo fastidió a éste. & .545 & & \\
\hline 21 & Que es asunto de ellos y no el mío. & .502 & & \\
\hline 25 & $\begin{array}{l}\text { No, porque uno no tiene que meterse en los juegos de los } \\
\text { demás. }\end{array}$ & .416 & & \\
\hline 27 & No, porque serían unos "sobones agua fiestas". & .527 & & \\
\hline 31 & $\begin{array}{l}\text { Que es solo responsabilidad del colegio, de los profesores o de } \\
\text { los adultos parar los malos tratos. }\end{array}$ & .325 & & \\
\hline 32 & Creo que no hay manera de como detenerlo. & .328 & & \\
\hline 34 & No lo sé, porque yo no me meto en esas cosas. & .355 & & \\
\hline 8 & Alegre, porque hacen cosas graciosas al compañero. & & .617 & \\
\hline 9 & Contento, porque ayudan a matar el aburrimiento en el aula. & & .625 & \\
\hline 15 & Les sigo la corriente y también fastidio. & & .543 & \\
\hline 16 & Me río, porque es muy chistoso. & & .502 & \\
\hline 30 & Nada, si solo es un juego. & & .425 & \\
\hline
\end{tabular}


Siento pena del (a) que ha sido molestada (o) e intento ayudarla.

11 Me molesto porque la hacen sentir mal.

Le digo al agresor(a) o a los agresores que dejen de molestarla.

17 Le riño al (a) abusiva para que la deje de molestar.

Que no deben comportarse de esa manera y deberían de

23 Que es algo injusto y que no debería de hacerlo.

28 Sí, y los malos tratos disminuirían.

29 Sí, intervenir para que paren de molestarla.

\begin{tabular}{cccc}
\hline Valor Eigen & 5.224 & 3.197 & 1.629 \\
\hline \% Varianza Explicada & 17.415 & 10.656 & 5.430 \\
\hline
\end{tabular}

\section{Denominación de factores}

El primer factor fue denominado "Espectador indiferente-culpabilizado", y engloba aquellos ítems que hacen referencia a quienes observan y no hacen nada; permanecen "neutrales" y pueden sentirse indiferentes con la situación, no dándole importancia en absoluto, con lo que toleran y se acostumbran a estas injustas situaciones. A veces se muestran nerviosos e incómodos/as, pero no hacen nada. No son responsables como agentes, pero sí como consentidores.

Las razones, que dan para no actuar y no decir nada suelen ser que: no quieren meterse en problemas, no se atreven a afrontar al abusador, impotencia frente a la situación, miedo a ser intimidadas, cobardía, concepto de lealtad y fidelidad mal entendidos, ideas erróneas respecto a ser cobarde; creer que acusar es un demérito en el código de los iguales.

El segundo factor resultante fue nombrado "Espectador Antisocial". Este factor, a diferencia del anterior, está constituido por aquellas expresiones que enuncian quienes no actúan directamente, pero refuerzan de alguna manera la conducta del abusador, por ejemplo, sonriendo o con gestos cómplices.

Están complacidos y a veces justifican el maltrato. Algunos pueden tener dudas y sentirse mal, pero se doblegan a las presiones del grupo o del abusador, ya que tienen miedo ellos mismos de ser objeto de burlas, intimidación o terminar fuera del grupo. A veces el abuso se hace en pandilla o grupo; son varios los que molestan y es difícil salirse de este grupo.

El tercer factor fue nombrado como "Espectador Prosocial", el cual está constituido predominantemente por quienes ayudan a la víctima y hasta pueden llegar a recriminar al agresor, verbal o gestualmente: "pata, te has pasado de la raya", "no seas abusiva". 
Se sienten afectados y suelen pedir ayuda y/o comunicárselo a los adultos.

\section{Análisis de la confiabilidad}

La estimación de la consistencia interna se realizó mediante el alfa ordinal (Dominguez, 2012; Elosua \& Zumbo, 2008) dado que las variables no son continuas, aspecto que es de suma importancia cuando se desea utilizar el Coeficiente Alfa de Cronbach; además de ello, la estructura del instrumento no es unidimensional, lo que desaconseja el uso del mencionado coeficiente.

Los hallazgos indican que el alfa ordinal fue de .707 para el factor "Espectador indiferente-culpabilizado"; de .674 para el factor "Espectador antisocial"; y de .801 para el factor "Espectador Pro social”.

En base a la configuración mostrada en la tabla anterior, se procedió a realizar un análisis confirmatorio de dicha estructura trifactorial, encontrándose que los datos se ajustan de modo aceptable al modelo de tres factores producto del análisis exploratorio: un GFI y AGFI mayores de .80, un RMSEA y RMR menores de .10, y finalmente, un CFI mayor de 70 (Ferrando \& Anguiano-Carrasco, 2010; Hu \& Bentler, 1999; Manzano \& Zamora, 2010; Ruiz, Pardo \& San Martín, 2010; Sánchez \& Sánchez, 1998), el cual aunque si bien no es una magnitud decisiva, es al menos satisfactoria (Hu \& Bentler, 1999).

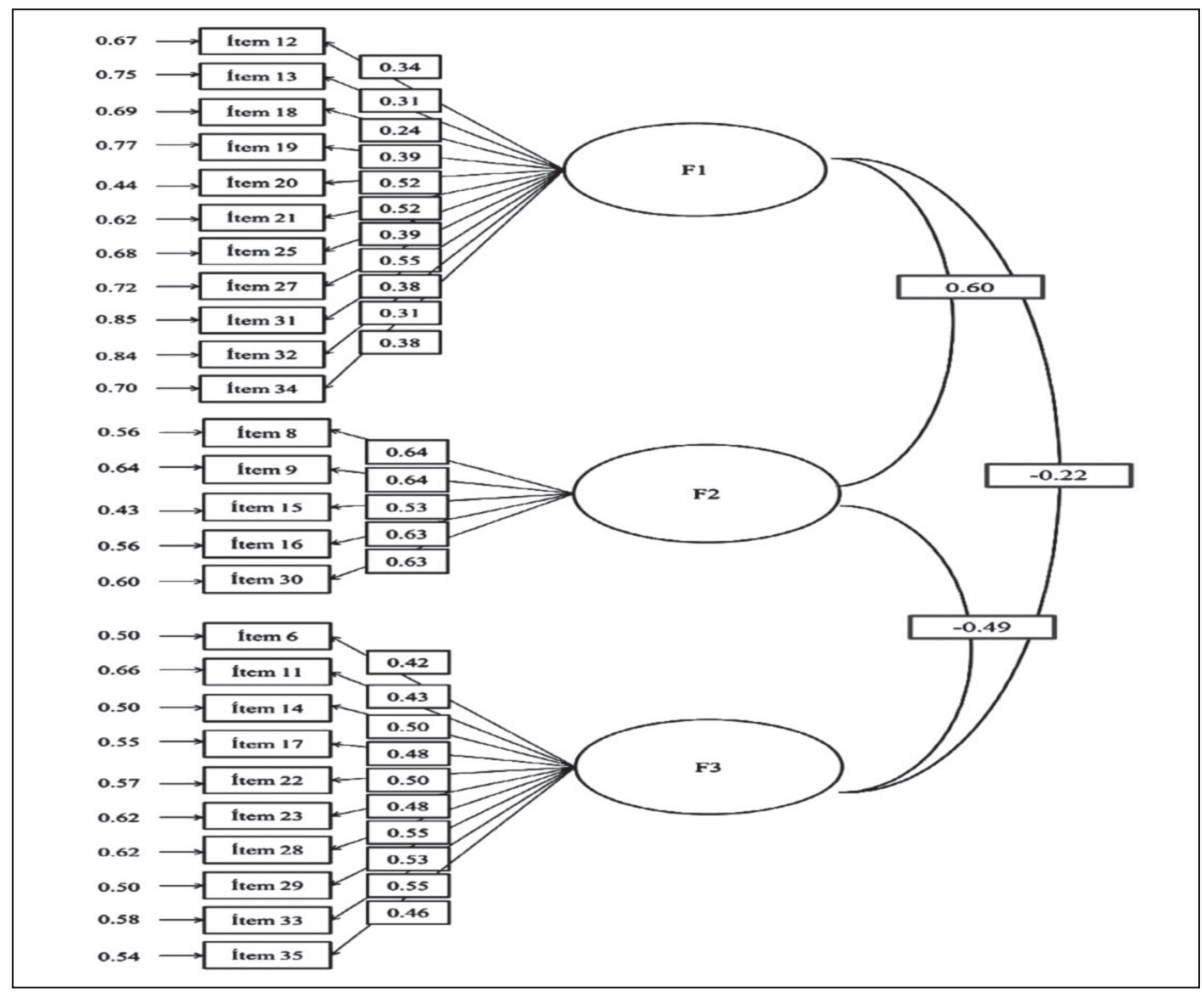

Gráfico 1. Modelo de Medición del Test de Espectadores de Violencia Entre Pares (TEVEP)-Revisado 


\section{Segundo análisis factorial confirmatorio}

Tabla 5. Índices de Ajuste del Test de Espectadores de Violencia Entre Pares (Tevep)- Revisado

\begin{tabular}{ccccccc}
\hline $\mathrm{X}^{2}$ & g.1. & CFI & GFI & AGFI & RMR & RMSEA \\
\hline $2065.3 *$ & 560 & .721 & .851 & .823 & .072 & .076 \\
\hline
\end{tabular}

$* \mathrm{p}<.001$

\section{Estadísticos descriptivos}

Finalmente, se presentan los estadísticos descriptivos de la escala elaborada.

Tabla 6. Estadísticos descriptivos del Test de Espectadores de Violencia Entre Pares (TEVEP) Revisado

\begin{tabular}{cccc}
\hline & F1 & F2 & F3 \\
\hline Media & 13.90 & 5.59 & 19.11 \\
Desviación Estándar & 5.21 & 3.49 & 5.44 \\
\hline
\end{tabular}

F1: Espectador indiferente-culpabilizado; F2: Espectador Antisocial; F3: Espectador Pro social.

\section{DISCUSIÓN}

El objetivo fundamental del trabajo fue realizar el análisis psicométrico de la escala, a fin de obtener evidencias de confiabilidad y validez que sustenten su uso en el contexto nacional, y permita la identificación del tipo de espectadores en la violencia entre pares.

Se inició el procesamiento con un análisis factorial confirmatorio debido a que su construcción fue guiada por supuestos previos (Ferrando \& Anguiano-Carrasco, 2010), los cuales son de índole teórica, y además se le considera una aproximación fuerte a la validez de constructo (Messick, 1995; Pérez-Gil, Chacón \& Moreno, 2000). De acuerdo con los resultados obtenidos, los datos no se ajustan al modelo de tres factores planteados inicialmente.

En tal sentido, los hallazgos no aportan a la consolidación del modelo de tres factores planteado inicialmente, y aunque otros indicadores como el GFI, AGFI, RMR y RMSEA presenten indicadores aceptables (Browne \& Cudeck, 1993; Ferrando \& Anguiano-Carrasco, 2010; Hair et al., 2004; Sánchez \& Sánchez, 1998), el Índice de Ajuste Comparativo (CFI) de Bentler (1990) está por debajo de lo aceptado que es .70 (Hu \& Bentler, 1999). Se debe considerar además que los resultados obtenidos dependen de la muestra analizada (Byrne, 2001).

En vista de esta situación, se llevó a cabo un análisis exploratorio, con el objetivo de descubrir la estructura subyacente a los datos. Dicho análisis dio cuenta de tres factores denominados "Espectador indiferente-culpabilizado", "Espectador antisocial" y "Espectador pro social", los cuales explican de manera conjunta el $33.50 \%$ de la varianza total de las puntuaciones. 
Con relación a la manera de organización de los ítems en los factores, no existieron reactivos complejos, es decir, con más de una carga factorial que lo relacionaba directamente con más de un factor. En tal sentido, esto apoya la independencia de los factores encontrados que hacen referencia a los tipos de espectadores.

Estos factores resultantes tienen relación con los aspectos teóricos ligados a los espectadores, en tanto son los que generalmente presencian, observan, conocen y/o sospechan de los actos de intimidación, están bien informados sobre la existencia de malos tratos entre compañeros, conocen quiénes son los/las maltratadores/as y quiénes las víctimas, y son ellos los primeros a los que el/la chico/a victimizado/a les contará su problema y les pedirá ayuda.

Finalmente, en un segundo análisis factorial confirmatorio, los datos se ajustaron de modo aceptable al modelo de tres factores producto del análisis exploratorio

Cabe mencionar que algunos reactivos quedaron fuera del análisis debido a que no cumplían los requisitos métricos mínimos considerados para ser admitidos. Quizá fue debido a que, al menos en la muestra estudiada, no representan indicadores de algún tipo de espectador, lo que puede estar estrechamente ligado a los niveles socioeconómicos predominantes en este trabajo que fueron el nivel bajo y nivel medio-bajo. En tal sentido, quedaría como recomendación ampliar la muestra a otros niveles socioeconómicos y estudiar el comportamiento de los cuatro reactivos eliminados.

Por último, los coeficientes de confiabilidad hallados reflejan una adecuada consistencia de los factores evaluados a nivel de coeficientes alfa ordinal.

La utilidad de esta escala abarca varias aristas. Los beneficios de identificar a los sujetos espectadores pro sociales con el fin de articular estrategias para combatir el acoso escolar a través de su participación activa, hacen, por ello, de alta prioridad de utilizar un instrumento confiable como el TEVEP. Pero también la identificación de los otros tipos de espectadores se puede considerar un objetivo positivo, porque mediante la escala se evidencian las aptitudes y habilidades faltantes en aquellos, pudiendo servir esos datos de base para la elaboración de un programa de entrenamiento que incida en su preparación pro social a fin de prevenir el bullying, como lo han relievado en su momento Monjas y Avilés (2006), Varela y Tijmes (2008), y Vaquero (2010). Creemos que la seriedad de los procedimientos con que se ha elaborado la versión final del Tevep, aquí presentada, garantiza su utilidad como un instrumento de uso preventivo en la escuela.

Entre las limitaciones cabe destacar que la muestra de estudio es más representativa de los escolares de nivel socioeconómico bajo, por lo cual los hallazgos presentados no pueden generalizarse a todos los niveles socioeconómicos. No obstante, dadas las fortalezas conceptuales y psicométricas de la escala, se sugiere iniciar estudios en centros de enseñanza de distintos niveles socioeconómicos, ya que es un aspecto relacionado con una convivencia saludable en la escuela y un aprender a vivir en democracia. 
Este último instrumento demostró ser útil en la detección de tipos de espectadores de violencia entre pares con las características de validez y confiabilidad adecuada para la población estudiada.

Queda pendiente para futuras investigaciones ampliar la muestra y obtener evidencias de validez convergente, considerando las correlaciones con otros constructos. Asimismo, se recomienda examinar las diferencias de acuerdo al sexo, estrato socioeconómico, etc.; son aspectos importantes a tener en cuenta.

\section{CONCLUSIONES}

1. La estructura teórica de tres tipos de espectadores se ve reflejada en la estructura factorial del instrumento, demostrando evidencias de validez de constructo.

2. Los indicadores no son concluyentes debido a las particularidades de la muestra, por lo cual pueden plantearse estudios posteriores que los superen.

3. La confiabilidad por el método de consistencia interna es adecuada.

4. El uso del instrumento permite identificar el tipo de espectador predominante, lo que facilitará el planteamiento de estrategias para el desarrollo de programas de promoción y prevención de la violencia entre pares en el ámbito escolar.

\section{REFERENCIAS BIBLIOGRÁFICAS}

Avilés, J. M. (2006). Bullying: el maltrato entre iguales. Agresores, víctimas y testigos en la escuela. Salamanca: Amarú Ediciones.

Avilés J. y Monjas C. (2005) Estudio de incidencia de la intimidación y el maltrato entre iguales en la educación secundaria obligatoria mediante el cuestionario CIMEI (Avilés, 1999). Anales de Psicología. Vol. 21, Nº 1, 27-41.

Bollen, K. (1989). Structural equations with latent variables. New York: Wiley.

Browne, M. W. y Cudeck, R. (1993). Alternative ways of assessing model fit. In: Bollen, K. A. y Long, J. S. (Eds.) Testing Structural Equation Models. pp. 136-162. Beverly Hills, CA: Sage

Byrne, B. (2001). Structural equation modeling with AMOS: Basic concepts, applications, and programming. Mahwah: Lawrence Erlbaum.

Collell, J. (2003) Adaptació del CSBS-P. Psicologia de la Salut UA B. Treball no publicat.

Dominguez, S. (2012). Propuesta para el cálculo del Alfa Ordinal y Theta de Armor. En Revista de Investigación en Psicología, 15(1), 213-217.

Elosua, P., y Zumbo, B. (2008). Coeficientes de fiabilidad para escalas de respuesta categórica ordenada. Psicothema, 20(4), 896-901.

Ferrando, P. y Anguiano-Carrasco, C. (2010). El análisis factorial como técnica de investigación en psicología. Papeles del Psicólogo, 31(1), 18-33. 
Ferrando, P. y Lorenzo-Seva, U. (1997). Procedimiento para evaluar el ajuste en análisis factorial exploratorio: el programa FICFACT. Revista Electrónica de Metodología Aplicada, 2(1), 50-64. Recuperado desde: http://www.unioviedo.es/reunido/index. $\mathrm{php} /$ Rema/article/view/9732/9478

Hair, J. F., Anderson, R. E., Tatham, R. L. \& Black, W. C. (2004), Análisis Multivariante. Madrid: Pearson.

Hu L. y Bentler P. (1999). Cutoff criteria for fit indexes in covariance structure analysis: Conventional criteria versus new alternatives. Structural Equation Modeling, 6, 1-55.

Manzano, A. y Zamora, S. (2010). Sistema de ecuaciones estructurales: una herramienta de investigación. México D.F.: Centro Nacional de Evaluación para la Educación Superior.

Messick, S. (1995). Standards of validity and the validity of standards in performance assessment. Educational Measurement: Issues and Practice, 15, 5-12.

Monjas M. y Avilés J. (2006). Programa de sensibilización contra el maltrato entre iguales. Junta de Castilla y León: Valladolid.

Olweus, D. (1998). Conductas de acoso y amenaza entre escolares. Madrid: Ediciones Morata.

Pérez, E. y Medrano, L. (2010). Análisis Factorial Exploratorio: Bases Conceptuales y Metodológicas. Revista Argentina de Ciencias del Comportamiento, 2(1), 58-66.

Pérez-Gil, J., Chacón, S. \& Moreno, R. (2000). Validez de constructo: el uso del análisis factorial exploratorio-confirmatorio para obtener evidencias de validez. Psicothema, $12(2), 442-446$.

Quintana P. y Ruiz S. (2013). Panorama de la investigación del Bullying y Cyberbullying en el Perú y el mundo. Bullying Opiniones Reunidas. Observatorio sobre la Violencia y Convivencia en la Escuela: Lima.

Quintana, A., Montgomery, W., Malaver, C. \& Ruiz S. (2011). Estilos explicativos y habilidades para la gestión de negociación de conflictos en adolescentes espectadores de violencia entre pares (Bullying). Revista IIPSI 14(1), 95-108.

Quintana, A., Montgomery, W., Malaver, C. (2009) Modos de afrontamiento y conducta resiliente en adolescentes espectadores de violencia entre pares. Revista IIPSI, 13(1), 153-171.

Ruiz, M., Pardo, A. \& San Martín, R. (2010). Modelos de Ecuaciones Estructurales. Papeles del Psicólogo, 31(1), 34-45.

Sánchez, E. y Sánchez, M. (1998). Los modelos de estructuras de covarianza como método de validación de constructo. En: Manzano, V. y Sánchez, M. (comps.). Investigación del Comportamiento. Innovaciones metodológicas y estrategias de docencia (pp. 101112). Sevilla: Instituto Psicosociológico Andaluz de Investigaciones.

Trautman, A. (2008). Maltrato entre pares o "bullying”. Una visión actual. Revista Chilena de Pediatría 79(1): 13-20.

Vaquero, M. (2010). PRECONCIMEI Cuestionario sobre Preconcepciones de Intimidación y Maltrato entre iguales. Materiales para la Convivencia Escolar. Recuperado el 12/01/2014 desde: http://convivencia.wordpress.com/2010/05/04/cuestionariopreconcimei-sobre-preconcepciones-de-intimidacion-y-maltrato-entre-iguales/ 
Varela, J. y Tijmes, G. (2008). Prevención de la Violencia Escolar: Paz Educa. Fundación Paz Ciudadana: Chile. Recuperado el 12/01/2014 desde: http://www.pazciudadana. cl/wp-content/uploads/2013/07/2008-12-01_Conceptos-N\%C3\%82\%C2\%BA-5Prevenci\%C3\%83\%C2\%B3n-de-la-violencia-escolar-Paz-Educa.pdf

\section{ANEXO 1}

\section{LA VERSIÓN FINAL DE LA PRUEBA}

¿Cómo te sientes cuando ves que algun@ de tus compañer@s molesta a otr@?

\begin{tabular}{|c|c|c|c|}
\hline & $\vec{w}$ & 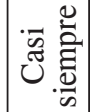 & \\
\hline
\end{tabular}

13. Siento pena de el(@) que ha sido molestad@e intento ayudarlo(@). N N CN CS S

14. Alegre, porque hacen las cosas graciosas al compañero.

$\mathrm{N} \quad \mathrm{CN} \quad \mathrm{CS} \quad \mathrm{S}$

15. Contento, porque ayudan a matar el aburrimiento en el aula. $\quad$ N $\quad$ CN $\quad$ CS $\quad$ S

16. Me molesto porque1@ hacen sentir mal.

$\mathrm{N} \quad \mathrm{CN} \quad \mathrm{CS} \quad \mathrm{S}$

¿Consideras que tus compañer@s deberían tratar de impedir que molestena otr@ compañer@

17. No, porque uno no tiene que meterse en los juegos de los demás. $\quad \begin{array}{llll}\mathrm{N} & \mathrm{CN} & \mathrm{CS} & \mathrm{S}\end{array}$

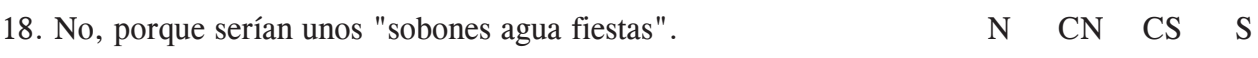

19. Sí, y los malos tratos disimularían. $\quad \mathrm{N} \quad \mathrm{CN} \quad \mathrm{CS} \quad \mathrm{S}$

$\begin{array}{lllll}\text { 20. Sí, intervenir para que paren de molestarl@. } & \mathrm{N} & \mathrm{CN} & \mathrm{CS} & \mathrm{S}\end{array}$

¿Qué piensas que se debe hacer para evitar que se sigan molestando unos a otros?

21. Nada, si solo es un juego.

$\mathrm{N} \quad \mathrm{CN} \quad \mathrm{CS} \quad \mathrm{S}$

22. Ques es solo responsabilidad del colegio, de los profesores o de los adultos parar los malos tratos.

23. Creo que no hay manera de como detenerlo.

24. Que alumnos, profesores y padres de familia debemos comprometernos a detener estos malos tratos.

25. No lo sé, porque yo no me meto en esas cosas.

26. Dialogar con 1@s chic@s y sus padres para que no molesten a sus compañer@s

$\mathrm{N} \quad \mathrm{CN} \quad \mathrm{CS} \quad \mathrm{S}$

$\mathrm{N} \quad \mathrm{CN} \quad \mathrm{CS} \quad \mathrm{S}$

N $\quad \mathrm{CN} \quad \mathrm{CS} \quad \mathrm{S}$

$\mathrm{N} \quad \mathrm{CN} \quad \mathrm{CS} \quad \mathrm{S}$

$\mathrm{N} \quad \mathrm{CN} \quad \mathrm{CS} \quad \mathrm{S}$ 


\section{TEVEP}

Con este cuestionario tratamos de ayudarle a mejorar el conocimiento sobre tus acciones y reacciones con los demas. También queremos saber como van las cosas y que podermos hacer entre todos para mejorarlas. Por esto te pedimos que contestes con sinceridad.

Lee las preguntas detenidamente. Para responder marca con un aspa (X) la frecuencia con que haces, piensas o sientes lo se afirma en cada frase.

$\begin{array}{ll}\mathrm{N} & \text { Nunca } \\ \mathrm{CN} & \text { Casi Nunca } \\ \mathrm{CS} & \text { Casi Siempre } \\ \mathrm{S} & \text { Siempre }\end{array}$

¿Si ves a alguien molestando a otr@ compañer@ en la escuela, que haces?

1. Me voy antes de que yo me convierta en lorna.

$\mathrm{N} \quad \mathrm{CN} \quad \mathrm{CS} \quad \mathrm{S}$

2. No hago nada para evitar que también a mí me molesten.

$\mathrm{N} \quad \mathrm{CN} \quad \mathrm{CS} \quad \mathrm{S}$

3. Le digo al agresor@o a los agresores que dejen de molestarl@.

$\mathrm{N} \quad \mathrm{CN} \quad \mathrm{CS} \quad \mathrm{S}$

4. Les sigo la corriente y también fastidio.

$\mathrm{N} \quad \mathrm{CN} \quad \mathrm{CS} \quad \mathrm{S}$

5. Me rio, porque es muy chistoso.

$\mathrm{N} \quad \mathrm{CN} \quad \mathrm{CS} \quad \mathrm{S}$

6.El riñoal@abusiv@para que 1@ deje de molestar

$\mathrm{N} \quad \mathrm{CN} \quad \mathrm{CS} \quad \mathrm{S}$

¿Qué piensas cuando ves que algun@ de tus compañer@s molesta a otr@?

7. Que debo evitar esas situaciones para no meterme en problemas.

$\mathrm{N} \quad \mathrm{CN} \quad \mathrm{CS} \quad \mathrm{S}$

8. Que si no1@s acusas no te van a molestar.

$\mathrm{N} \quad \mathrm{CN} \quad \mathrm{CS} \quad \mathrm{S}$

9. Que se lo merece porque seguro antes lo fastidió a éste.

$\mathrm{N} \quad \mathrm{CN} \quad \mathrm{CS} \quad \mathrm{S}$

10. Que es asunto de ellos y no el mío.

$\mathrm{N} \quad \mathrm{CN} \quad \mathrm{CS} \quad \mathrm{S}$

11. Que no deben comportarse de esa manera y deberían de respetar a sus compañeros.

$\mathrm{N} \quad \mathrm{CN} \quad \mathrm{CS} \quad \mathrm{S}$

12. Que es algo injusto y que no debería hacerlo.

$\mathrm{N} \quad \mathrm{CN} \quad \mathrm{CS} \quad \mathrm{S}$ 